Int. J. Odontostomat., 8(1):167-173, 2014.

\title{
Afectación de la Salud Oral en Niños que Padecen Maltrato Infantil: Reporte de Caso
}

\author{
Impact of Oral Health in Children with Child Abuse: A Case Report
}

Alejandra Requena Mendoza*; Norma Leticia Robles Bermeo* \& Edith Lara Carrillo**

REQUENA, M. A.; ROBLES, B. N. L. \& LARA, C. E. Afectación de la salud oral en niños que padecen maltrato infantil: Reporte de caso. Int. J. Odontostomat., 8(1):167-173, 2014.

RESUMEN: El maltrato infantil es una patología de la cual se tienen registros desde las civilizaciones antiguas como la griega y egipcia; pero es hasta 1924 que se firma la declaración de Ginebra: Derechos de la infancia, siendo el IX derecho: No al Maltrato. En México se tiene registro de maltrato infantil desde hace 20 años. Se ha tipificado el maltrato infantil en dos grandes rubros: activo y pasivo, la negligencia odontológica es clasificado como maltrato pasivo, por lo tanto el odontólogo (en especial el Odontopediatra) debe prestar atención al hacer la inspección clínica y elaboración de historia clínica del paciente, para encontrar indicios de maltrato infantil y llevar a cabo la atención de estos pacientes con adecuado manejo de su conducta, la cual puede ser variable dependiendo el tipo de maltrato que ha sufrido. Las consecuencias del maltrato en cavidad oral son múltiples y pueden manifestarse a corto, largo y mediano plazo. En este artículo se presenta un caso clínico de maltrato por abandono, negligencia odontológica y las consecuencias que éste tuvo en la salud oral y general de la paciente.

PALABRAS CLAVE: maltrato a los niños, salud oral, odontología pediátrica, negligencia infantil.

\section{INTRODUCCIÓN}

La clínica de Atención Integral al Niño Maltratado del Instituto Nacional de Pediatría define el maltrato infantil como toda agresión física, sexual, psicológica o negligencia intencional contra una persona menor de edad, en cualquier etapa de la vida, que afecta su integridad biopsicosocial, realizada habitual u ocasionalmente, dentro o fuera del hogar, por una persona, institución o sociedad en función a su superioridad física, intelectual o económica (Loredo-Abdalá, 2008). En el libro "Niños Maltratados", se define como "La acción, omisión o trato negligente, no accidental, que prive al niño de sus derechos y su bienestar, que amenace o interfiera su ordenado desarrollo físico, psíquico y/o social, cuyos autores pueden ser personas, instituciones o la propia sociedad" (Medrano \& Perona, 2010).

Según la Ley, se considera maltrato infantil todo acto realizado a menores de 18 años cuando su salud física o mental, o su seguridad, están en peligro, ya sea por acciones y omisiones llevadas a cabo por los padres u otras personas responsables de su cuidado produciendo, el maltrato por acción, omisión o negligencia (Ferro et al., 2010).

El maltrato infantil es una patología que se conoce como el síndrome del niño maltratado. Actualmente se ha reconocido como un problema de rezago, ya que este padecimiento es casi tan antiguo como la humanidad (del Bosque-Garza, 2003).

El primer caso de maltrato infantil se reportó el año 1874, en la ciudad de Nueva York, y fue llevado a la Sociedad Americana de Prevención de la Crueldad a los Animales. En 1924, se estableció la Declaración de Ginebra: Derechos de la infancia, siendo el IX derecho: No al Maltrato (Medrano \& Perona, 2010). El Dr. Jaime Mercovich, hace 20 años señalaba la existencia de niños maltratados en México y Lloyd deMause asevera: "mientras más se retrocede en el pasado, más expuestos están los niños a la muerte 
violenta, abandono, golpes, terror, y abusos sexuales" (del Bosque-Garza). En 1962 se fundó la Oficina Federal del Niño del Departamento de Salud, Educación y Bienestar, que publicó la guía legislativa "Principios y terminología sugerida para la legislación sobre denuncia de niños maltratados". A partir de 1980 se reconoce el maltrato infantil como prioridad en la sociedad; ésta no distingue raza, sexo, ni condición social (Medrano \& Perona). A partir del año 1999, la OMS considera al maltrato infantil como un problema de salud pública, al considerar el impacto físico y emocional que la agresión ocasiona al menor, el choque emocional y económico que causa a la familia y la repercusión económica y social que produce en la comunidad (Loredo-Abdalá).

Etiología del maltrato infantil. El maltrato infantil no es un hecho aislado, es un proceso que está determinado por la intervención de factores sociales, familiares, ambientales y del propio niño, que interaccionan de forma dinámica y pueden coincidir en una misma familia y en un determinado momento (Medrano \& Perona). Las causas más comunes de maltrato infantil son un modelo psicológico, economía, desintegración familiar, inmadurez emocional y baja autoestima de parte de los padres o cuidadores, falta de preparación para atender a niños discapacitados, desconocimiento de las leyes que protegen al niño, conductas aprendidas y disciplina a través del maltrato (Ferro et al.).

Tipos de maltrato infantil. El maltrato Infantil se subdivide en dos grandes grupos:

\section{A. Pasivo:}

a. Abandono físico: cuando las necesidades físicas básicas del menor no son atendidas por ningún miembro que conviva con él.

b. Abandono emocional: falta de respuesta a las necesidades de contacto físico y caricias del menor y la indiferencia al estado de ánimo del niño.

c. Omisión-Negligencia: un adulto permite conscientemente que el niño sufra o cuando no se satisfacen las necesidades esenciales para su desarrollo.

Negligencia Odontológica: definida por la Academia Americana de Odontología Pediátrica como la falta deliberada, por parte de padres o tutores, de la búsqueda o seguimiento del tratamiento necesario para asegurar un nivel de salud oral esencial para desarrollar una función adecuada y garantizar la ausencia de dolor e infección orales y otras condiciones de la cavidad oral y estructuras de soporte que causen una mala alimentación, retardo de crecimiento y desarrollo, dificultando la vida diaria del menor.

\section{B. Activo:}

d. Abuso físico: lesión corporal que el padre, cuidador o tutor cause a un niño intencionalmente.

e. Abuso sexual: explotación mediante cualquier acto sexual que va desde exposición indecente, tocamiento indebido, violación, prostitución, rapto, incesto, pornografía y comercio sexual.

f. Abuso emocional: patrón de comportamiento que retrasa y deteriora el desarrollo psíquico del niño y su autoestima; va desde la hostilidad verbal, acoso, insulto, menosprecio, sometimiento, dominación, abuso pedagógico (niños con excesivas obligaciones pedagógicas), evitando el desarrollo normal y vida social del niño.

g. Explotación laboral: acciones como la mendicidad, venta ambulante, etc. (Loredo-Abdalá; MoranteSánchez \& Kanashiro-Irakawa, 2009; Ferro et al.; Medrano \& Perona).

Tanto el maltrato como el abandono están relacionados con consecuencias negativas a corto y largo plazo en la salud mental y física del niño, en su capacidad de aprendizaje, en su rendimiento académico y en su desarrollo social y de comportamiento (del Bosque-Garza).

\section{Indicadores del maltrato infantil en niños}

Conductuales: ausencias reiteradas a clase, bajo rendimiento escolar, dificultades de concentración, depresión constantes o conductas auto-agresivas, ideas suicidas, docilidad excesiva, actitud evasiva o defensiva frente a los adultos, la búsqueda intensa de expresiones afectuosas, actitudes o juegos sexualizados para su edad, intrusión, ansiedad, trastornos de sueño, baja autoestima, conductas adictivas, evidencia de "inversión de papeles".

Físicos: alteración de factores normales de crecimiento y desarrollo, falta de higiene y cuidado corporal, marcas de castigos corporales, accidentes frecuentes, embarazo precoz, vestidos inapropiados para la temporada (camisa de manga larga en días calurosos).

Indicadores oro-faciales. Signos y síntomas en la cavidad oral: presencia de petequias en el paladar, 
desagarro en el frenillo labial y lingual, retraso para emprender el tratamiento, caries en número abundante, abscesos repetidos, granulomas, ausencia de dientes, enfermedades periodontales (Ferro et al.; Medrano \& Perona).

Otras lesiones: áreas de alopecia traumática, traumatismos y hemorragias cráneo-encefálicos, hematomas, desgarros, arañazos, equimosis, mordiscos en la cara, estigmas ungueales en el cuello, quemaduras, laceraciones, y contusiones en la cavidad oral (Oliván Gonzalvo, 2002).

Actitud del odontólogo ante un niño matratado. Entre el 60 y $70 \%$ de los casos, las victimas presentan lesiones de cabeza, cara, cuello y boca (Flores- Ríos \& Monter García, 2012). El odontólogo debe estar alerta ante posibles signos y síntomas que pueden verse en la cavidad oral; el diagnóstico comienza con una buena historia clínica, cuando se examina a un paciente con lesiones potencialmente no accidentales como son quemaduras, lesiones en mucosas, mordeduras, laceraciones, luxaciones y fracturas dentales, rotura de frenillos, eritema, petequias, fracturas radiculares, etc. (Ferro et al.) Además el odontólogo obtendrá una impresión general de niño: higiene, desarrollo, estatura, vestimenta, relación con los padres, marcas inusuales en la piel, limitación de movimientos; todo esto en busca de un exámen completo y sistemático.

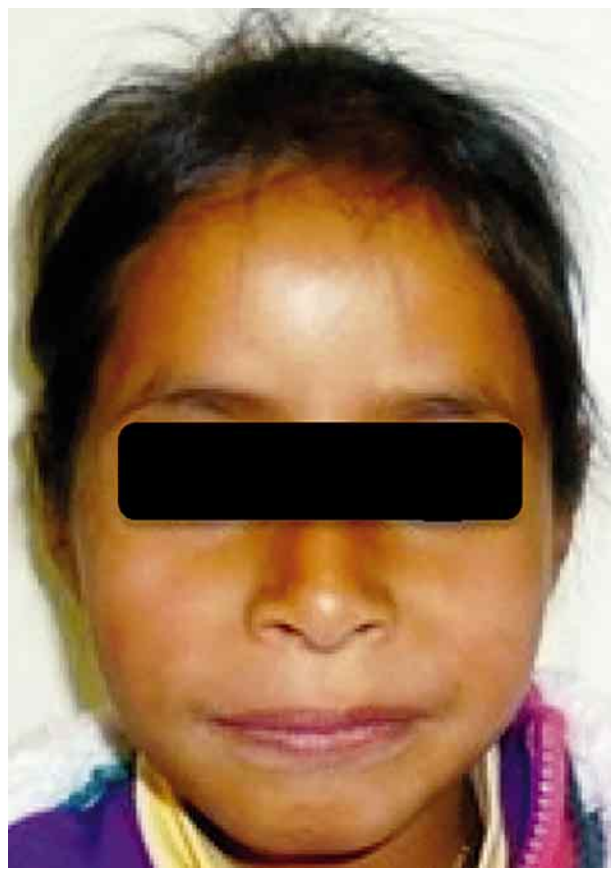

Fig. 1. Paciente sexo femenino de 9 años de edad dolicofacial, simétrica que presenta manchas blanquecinas en las mejillas.
En México la legislación que rige sobre maltrato y violencia infantil son: Ley para la protección de niños, niñas y adolescentes, vigente desde 2005; en el estado de México Ley para la prevención y atención la Violencia familiar, Ley para la protección de los derechos de las niñas, niños y adolescentes, vigentes desde 2004. El maltrato infantil deja huellas físicas observables así como secuelas emocionales que provocan un impacto en el desarrollo social, educativo y emocional del niño; con consecuencias a mediano y largo plazo (López-Orozco, 2010).

El objetivo de este artículo es conocer las repercusiones que el abandono deja en el aparato estomatognático de un niño, provocadas por la falta de atención física, médica, educativa y emocional, que tendrán consecuencias en su desarrollo.

\section{REPORTE DE CASO}

Se presenta el caso de una niña de 9 años de edad, que acude a consulta odontología para revisión y tratamiento. Al interrogatorio el padre refiere que la niña se encuentra bajo su custodia legal y habitando en un Internado-Escuela, de lunes a viernes permitiéndole visitas y convivencia con el padre los fines de semana. Anteriormente la paciente vivía bajo la custodia de la madre teniendo alimentación a base de leche en biberón hasta la edad de 6 años y su alimentación sólida fueron frijoles y tortilla hasta la edad de 8 años. Actualmente se encuentra bajo régimen nutricional supervisado por nutriólogo dentro del internado. El padre refirió que la paciente tiene 3 hermanos menores que se encuentran bajo custodia legal del DIF en el Estado de Zacatecas, y que muestran las mismas condiciones orales y físicas que la paciente.

A la inspección clínica se observa un peso de $18,5 \mathrm{Kg}$ y talla de $1,20 \mathrm{~m}$, que se encuentra por debajo a lo esperado para su edad (Fig. 1). El motivo de consulta referido por el padre, es que la dentista de la escuela la remite a la Clínica de la Especialidad de Odontopediatría en la Facultad de Odontología de la Universidad Autónoma del Estado de México, debido a la alta incidencia de caries y presencia de múltiples focos infecciosos. A la inspección clínica, se observaron múltiples lesiones cariosas de grado variable, abscesos periapicales crónicos con presencia de fístula; gingivitis generalizada y presencia de cálculo dental, malposiciones y maloclusiones dentarias (Fig. $2 \mathrm{~A}$ y $\mathrm{B}$ ). 


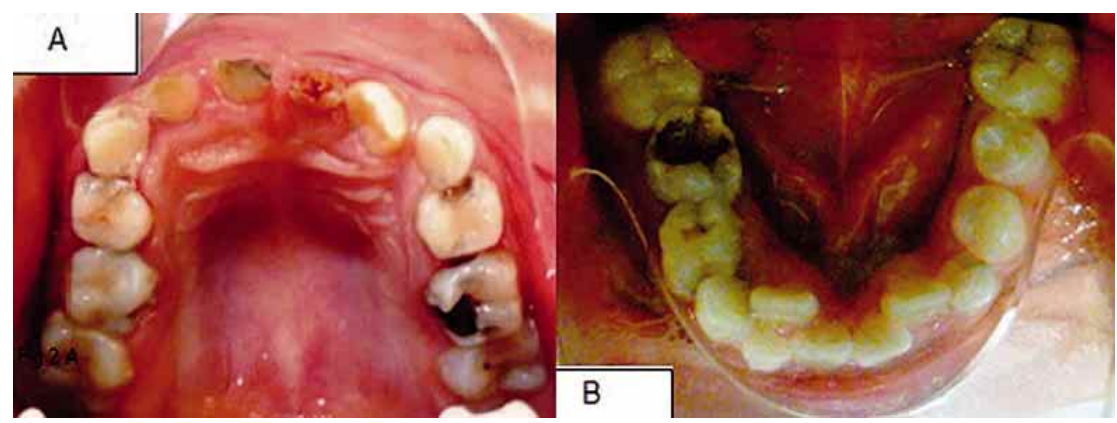

Fig. 2. A. Arco cuadrangular, paladar medio, mucosas rosa pálido, dentición mixta, presencia de múltiples lesiones cariosas de grado variable. B. Arco en herradura, mucosa rosa pálido, piso de boca permeable, dentición mixta, con apiñamiento y malposiciones dentales, presencia de múltiples lesiones cariosas de grado y extensión variable.

Mediante el estudio radiográfico se observa una malposición de los dientes 11 y 21 que presentan retraso en erupción, y persistencia de los dientes 52 y 61 , y lesiones radiolúcidas extensas. Los dientes 85 y 84 presentan poco soporte óseo y lesiones radiolúcidas de gran extensión (Fig. 3).

Se pidió interconsulta con el servicio de Pediatra en el Hospital para el Niño del Instituto Materno Infantil del Estado de México, para la valoración del estado general de salud de la paciente; se recibe hoja de contra-referencia en la cual se diagnostica anemia leve y se da tratamiento con suplementos alimenticios; permitiéndose iniciar el tratamiento dental. Las consultas se iniciaron con modelaje para el manejo de conducta con la paciente, quien respondía vagamente a las indicaciones y preguntas que se le realizaban; se contaba con su cooperación pero se quedaba indiferente durante los tratamientos, presentando poco interés respecto a lo que ocurría durante la consulta odontológica.

Como tratamiento se inició un plan preventivo: control de placa dentobacteriana, técnica de cepillado, detartraje, profilaxis dental; posteriormente extracción de los dientes $51,61,74$ y 75 por diagnóstico absceso dental crónico con presencia de fístula y movilidad grado 4, pulpectomía del diente 65 por absceso apical crónico con presencia de fístula y movilidad grado 3 . Mediante el estudio radiográfico periapical en el cual se observa dentición mixta, presencia de gérmenes dentarios, diente 21 en posición incorrecta, y persistencia de los dientes 52,51, 61 y 62 , con zonas radiolúcidas extensas. Dientes 34,35 y 36 en erupción, apiñamiento de los dientes $32,31,41$ y 42 . Zona radiolúcida a nivel óseo en los dientes 84 y 85 , presencia de dientes en desarrollo intraóseo (Fig. 4A y B).

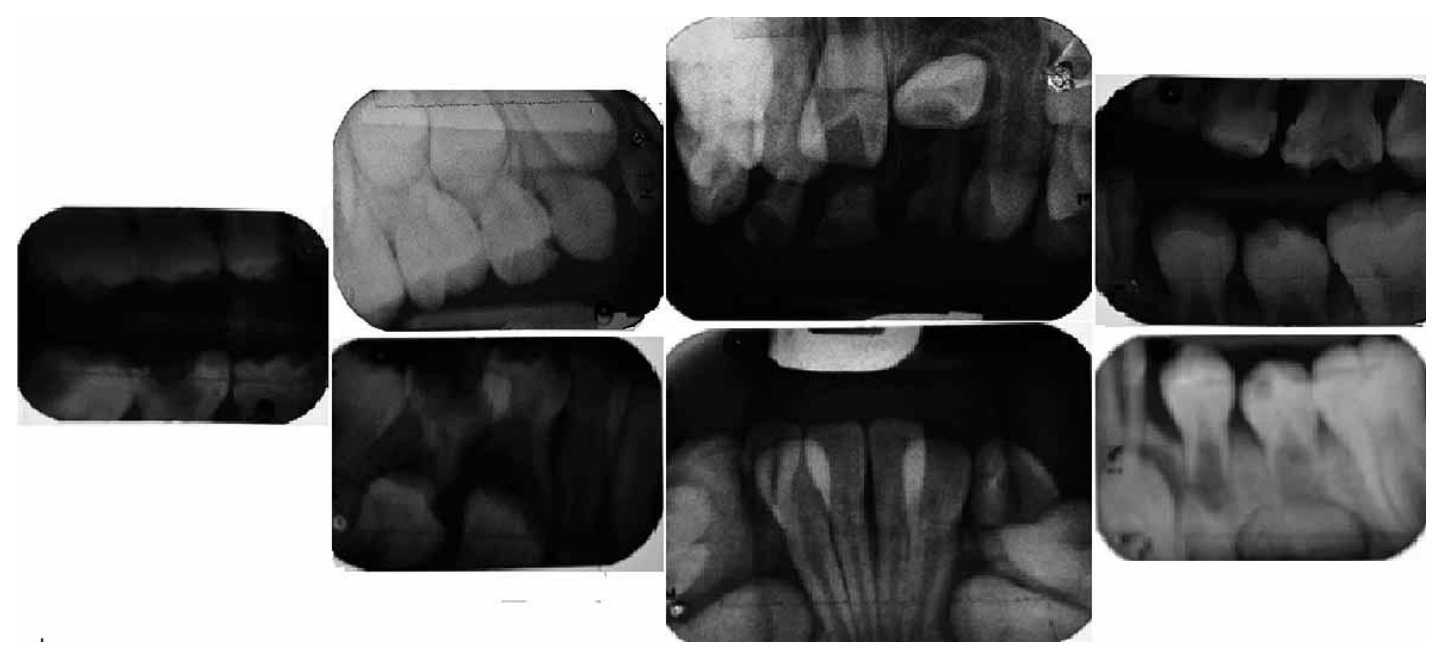

Fig.3. Estudio radiográfico periapical de la paciente, donde se observa el estado de los dientes temporales y definitivos. 


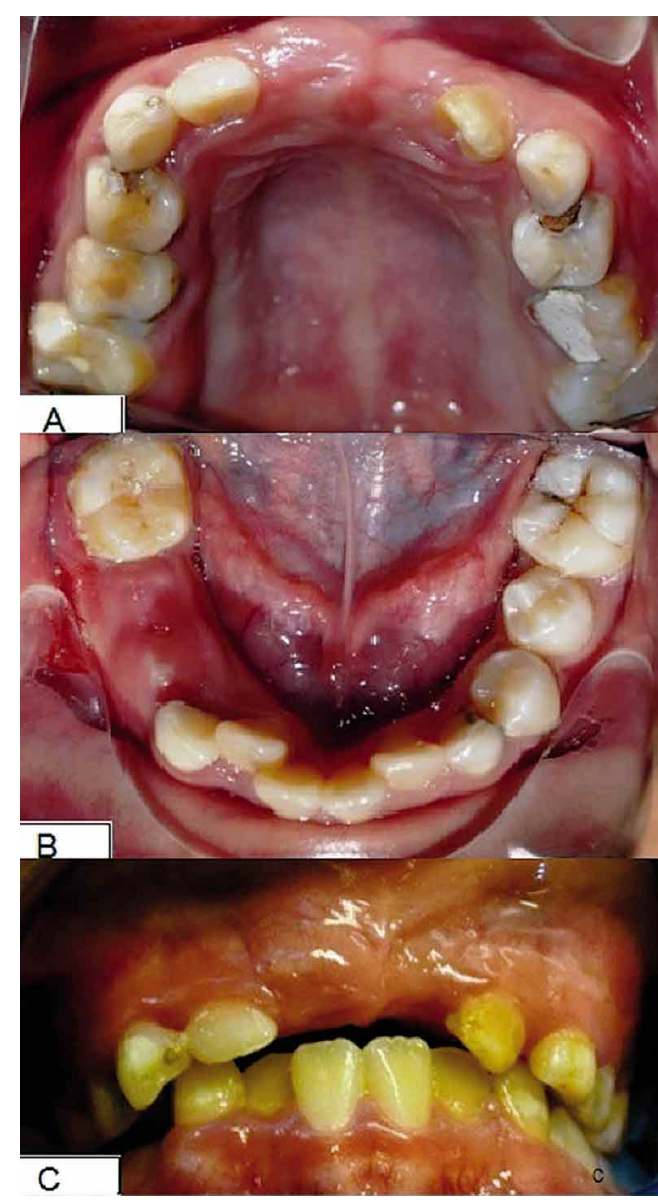

Fig. 4. A. Sextante anterosuperior muestra correcta cicatrización posterior a las extracciones realizadas, diente 12 se encuentra en erupción, curación temporal del diente 65. B. Comienzo de la erupción del diente 44 posterior a la extracción de los dientes 84 y 85 . C. Pérdida de la dimensión vertical posterior a la extracción de los dientes 52, 51, 61 y 62 . Acumulo de placa dentobacteriana e inflamación gingival.
Se continuó con refuerzo de plan preventivo debido al acúmulo de placa dentobacteriana (Fig. 4C). En cada consulta se realizó control deplaca dentobacteriana y reforzamiento de técnica de cepillado, lo que redujo el índice de placa hasta un $20 \%$.

Durante el manejo de la conducta se interactuó paulatinamente con la paciente mientras transcurrían las citas hasta que se logró una relación de confianza con ella y una comunicación más efectiva que benefició el avance del tratamiento dental. Conforme avanzaron las citas, mediante modelamiento y refuerzo positivo, se logró cooperación total de la paciente y una relación que facilito la comunicación con ella; quien mostraba mayor interés en el tratamiento dental y motivación en la realización del cepillado dental mejorando el cuidado de su salud oral.

Se pidieron estudios radiográficos (radiografía panorámica y radiografía lateral de cráneo) (Fig. 5A y B) para valorar el estado dentario y las relaciones dento-esqueléticas. Se confirmaron malposiciones de los dientes 11 y 21 que dificultaran su erupción correcta.

Se dio de alta a la paciente habiéndola rehabilitado por completo con los siguientes tratamientos: terapias pulpares (pulpotomías y pulpectomía), coronas de níquel cromo, resinas, sellado de fosas y fisuras, profilaxis y aplicaciones tópicas de fluoruro. Se observan encía color rosa coral que ha mejorado aspecto después de un plan de prevención con técnica de cepillado y controles de placa, dientes $12,22,35,34$ y 44 en erupción, diente 22 con hipoplasia y en proceso de erupción (Fig. 6A y B).

Posteriormente la paciente fue remitida a la Clínica del Posgrado de Ortodoncia de la Facultad de Odontología de la Universidad Autónoma del Estado de México, para su tratamiento ortodóncico.

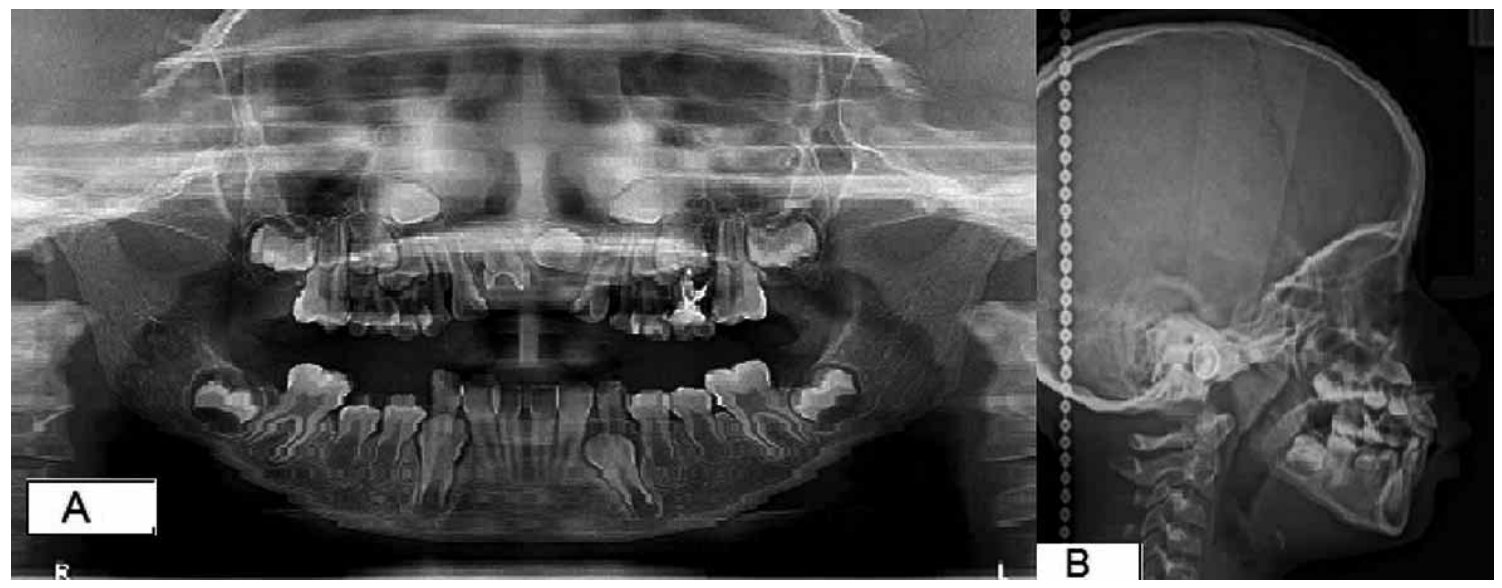

Fig. 5. A. Radiografía Panorámica. B. Radiografía Lateral. 


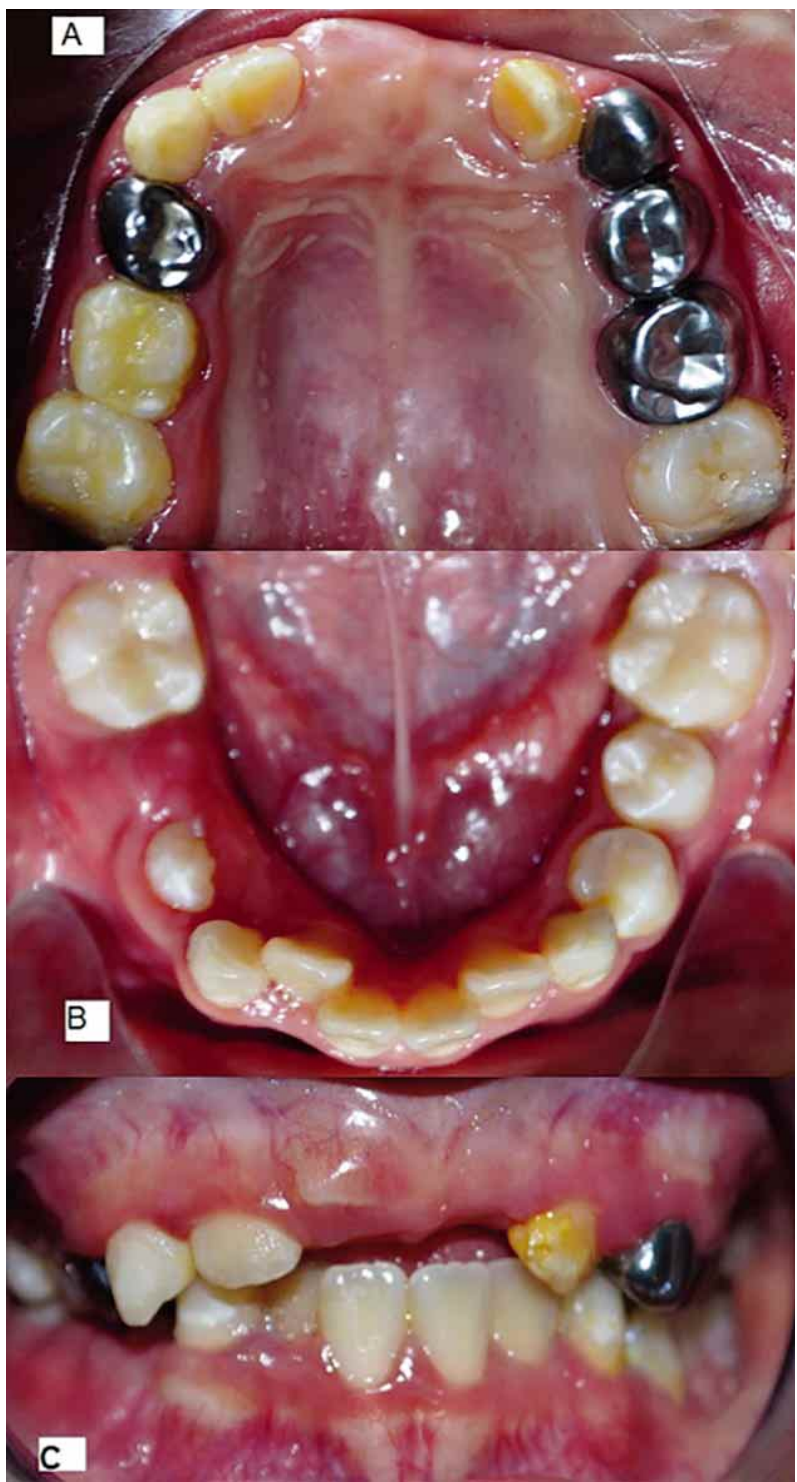

Fig. 6. A. Fotografías finales del maxilar y sus dientes intervenidos; nótese la presencia de coronas metálicas para mantener los dientes temporales. B. Restauraciones de resina finales y sellado de surcos y fosuras en dientes definitivos. C. Fotografía de máxima intercuspidación posterior a la rehabilitación oral.

\section{DISCUSIÓN}

El maltrato infantil se define como toda agresión física, sexual, psicológica o negligencia intencional contra una persona de menor edad, en cualquier etapa de la vida, que afecta su integridad biopsicosocial (Loredo-Abdalá). El maltrato infantil se ha tipificado como "Activo" y "Pasivo", en este último se encuentra la Omisión-Negligencia que es cuando un adulto permite conscientemente o no que el niño sufra o cuando no se satisfacen las necesidades esenciales para su desarrollo; dentro de estas necesidades esenciales se encuentra la salud oral del menor, la cual al verse afectada tiene consecuencias su desarrollo integral.

La negligencia odontológica (Medrano \& Perona) en el caso reportado, se muestra como la omisión en la atención odontológica de la paciente tuvo consecuencias en su desarrollo; con una malposición dental, pérdida dental prematura con la erupción precoz de los dientes permanentes; malposición de los dientes 11 y 21 los cuales aún no erupcionan, así como el apiñamiento dental e hipoplasia del diente 22; todas estas anomalías contribuyen a que el desarrollo general de la paciente se vea disminuido.

El maltrato infantil "Pasivo" al igual que el maltrato infantil "Activo" tiene repercusiones tanto físicas y psicológicas en el menor; el odontólogo tiene la obligación mostrarse atento al estado físico general del paciente y brindarle la atención oportuna que contribuya en la mejora del desarrollo adecuado del menor que ha sido víctima de maltrato.

El maltrato infantil deja huellas físicas observables así como secuelas emocionales que provocan un impacto en el desarrollo social, educativo y emocional del niño; teniendo consecuencias a mediano y largo plazo (López-Orozco). En nuestro reporte, las repercusiones de el maltrato en forma de abandono que sufrió la paciente dejó secuelas que le afectarán a mediano y largo plazo, tanto físicas como emocionales, ya que el hecho de tener una salud oral deficiente que se ha reflejado en su crecimiento y desarrollo, manifestándose en la dificultad para comer por la severidad de las lesiones cariosas y procesos infecciosos, así como el bajo rendimiento escolar referido por los profesores de la menor y manifestado por el padre.

REQUENA, M. A.; ROBLES, B. N. L. \& LARA, C. E. Impact of oral health in children with child abuse: A case report. Int. J. Odontostomat., 8(1):167-173, 2014.

ABSTRACT: Child abuse is a disease for which there are records from ancient civilizations like the Greek and Egyptian, but until 1924 when the Geneva Declaration was signed: Children's rights: No to Abuse. In Mexico records of child abuse have been kept for the last 20 years. It has classified child abuse into two major categories: active and passive, dental malpractice liability is classified as child abuse therefore the dentist (especially the pediatric dentist) should pay attention to 
realize a clinical inspection and preparation of patient history, for signs of child abuse, and carry out the care of these patients with adequate management of their behavior, which can vary depending on the type of abuse the child has suffered. The consequences of abuse in oral cavity are multiple and can occur at short, medium and long term. This article presents a case of abuse by dental neglect and the consequences that this has on oral health, and therefore on the general health of the patient.

KEY WORDS: child abuse, oral health, dentistry pediatric, child neglect, child maltreatment.

\section{REFERENCIAS BIBLIOGRÁFICAS}

Del Bosque-Garza, J. IV. Historia de la agresión a los niños. Gac. Med. Méx., 139(4):368-70, 2003.

Ferro, T. M. J.; Maldonado, A.; Montiel, Y. \& Rivas, M. A. Implicaciones psicológicas del paciente odontológico con maltrato infantil. Revista Latinoamericana de Ortodoncia y Odontopediatría. 2010. Disponible en: http:// www.ortodoncia.ws/publicaciones/2010/pdf/art3.pdf

Flores-Ríos, J. D. \& Monter García, M. A. Indicadores de Maltrato infantil: reporte de un caso. Rev. Acad. Mex. Odont. Ped., 24(2):96-100, 2012.

López-Orozco, E. Maltrato infantil. En: González Hernández, E. Revista del Tribunal Superior de Justicia del Distrito Federal. México D.F., Tribunal superior de justicia del Distrito Federal, 2010. pp.164-6.

Loredo-Abdalá, A. Maltrato infantil: consideraciones básicas, para el diagnostico de las formas más preponderantes. Acta Pediatr. Mex., 29(5):255-61, 2008.

Medrano, G. G. \& Perona, M. G Maltrato infantil: una realidad muy cercana, ¿Cómo debemos actuar los odontólogos? Odontol. Pediatr., 9(1):78-94, 2010.

Oliván Gonzalvo, G. Indicadores de Maltrato Infantil. Guías Clínicas, 2(44), 2002. Disponible en: http:// www.saludmentalextremadura.com/fileadmin/documentos/Guias_de_Practica_Clinica/FISTERRA/Maltrato.pdf
Dirección para Correspondencia:

R EOP. Alejandra Requena Mendoza

Especialidad en Odontopediatría

Facultad de Odontología

Universidad Autónoma del Estado de México

Paseo Tollocan, Esq. Jesús Carranza.

Colonia. Universidad. CP 50130. Toluca

MÉXICO

Email: alejandrarequenamendoza@yahoo.com.mx

Recibido : 04-12-2013

Aceptado: 22-02-2014 